\title{
Strategy shift affordance and strategy choice in young and older adults
}

\author{
DAYNA R. TOURON and CHRISTOPHER HERTZOG \\ Georgia Institute of Technology, Atlanta, Georgia
}

\begin{abstract}
When skill acquisition involves a shift in strategy (such as from rule-based to retrieval-based processing), older adults typically shift later in practice than young adults do. We observed the shift from scanning-based to memory-based processing in a noun pair learning task. Young and older adults were trained in conditions in which the relationship between memory load and scanning load was manipulated by making the strategy shift more or less beneficial. Older adults in a condition with high shift affordance shifted to memory retrieval more fully and more rapidly than did older adults in conditions with lower shift affordance. Reluctance to rely on memory retrieval was related to metacognitive reports of memory confidence. The present study indicates that age differences in skill acquisition reflect qualitative age differences in strategy choice in addition to quantitative age differences in component task processes.
\end{abstract}

Theories of skill acquisition typically characterize the transition from novice to skilled performance as involving both qualitative shifts in how information is processed and improvements in processing efficiency. Fluent performance can often be achieved through direct and rapid retrieval of stored memory representations (Ericsson \& Charness, 1994; Logan, 1988; Reder \& Ritter, 1992; Shiffrin \& Schneider, 1977). If a cognitive task affords the use of memory retrieval to drive fluent processing, skill acquisition is predominantly governed by a shift from slow rulebased performance to faster retrieval-based performance.

Logan's (1988; see also Wenger, 1999) instance model accounts for skill acquisition in terms of a horse race between the rule process and the retrieval process. At a given point in training, the process with the fastest latency wins the race. Rickard's (1997) component power law (CMPL) model argues instead for a strategy choice mechanism as the fundamental basis for strategy shift. The strategy shift does not stem from faster completion of the retrieval process, relative to the rule process, but instead reflects the occurrence of strategy selection early in item processing. If retrieval is selected, the rule process is not executed, and vice versa. Hence, strategy selection precedes retrieval success. Work by Reder and colleagues (e.g., Reder \& Ritter, 1992; Schunn, Reder, Nhouyvanisvong, Richards, \& Stroffolino, 1997) has shown that individuals can make a rapid decision about whether they know an answer on the basis of problem familiarity and can then base subsequent

This research was supported by the National Institute on Aging Grant NIA 23T2 AG00175-11 and by Cognitive Aging Training at the Georgia Institute of Technology Grant NIA R37 AG13148 to C.H. Correspondence concerning this article should be addressed to D. R. Touron, Department of Psychology, Appalachian State University, Boone, NC 28608 (e-mail: tourond@appstate.edu). strategy selection on that feeling of knowing (FOK), irrespective of whether a candidate answer has been retrieved from memory.

No strategy shift need occur when the rule process is sufficiently fast (Bourne, Healy, Parker, \& Rickard, 1999; Cerella, Green, \& Hoyer, 1999). Hence, strategy shift can be conceived of as a transition that occurs when the cost of strategy shift (the difficulty of memorization) is warranted by the benefits (decreases in processing demands and increases in processing efficiency; see, e.g., NavehBenjamin, Craik, Guez, \& Dori, 1998). Irrespective of any objective costs and benefits, subjective perceptions of costs and benefits might also influence shift. We refer to the relative costs and benefits for the retrieval strategy as strategy shift affordance; experimental manipulations that increase the relative benefit of a retrieval strategy over an algorithmic strategy can be said to increase the task's affordance for strategy shift.

The present study evaluated age differences in the timing of the strategy shift in a skill acquisition task. A body of research suggests that older adults can acquire new skills but typically require greater amounts of training to do so (see Bosman \& Charness, 1996). Age differences in rates of skill acquisition may be related to strategy shift patterns. Older adults typically either continue to use a rule-based strategy or shift to retrieval later in training than younger adults do (Charness \& Campbell, 1988; Jenkins \& Hoyer, 2000; Rogers, Hertzog, \& Fisk, 2000; Touron, Hoyer, \& Cerella, 2001).

A critical issue is how this slowing in rates of skill acquisition should be interpreted. Slower strategy shift in older adults could be attributed to age-related learning deficits (e.g., Salthouse, 1994; see Kausler, 1994, for a review) - that is, older adults with learning deficits would be forced to shift more slowly to a retrieval strategy. Al- 
ternatively, age differences in strategy selection preferences could exaggerate the role of learning deficits in the slowing of strategy shift. Individuals who are motivated to memorize information in order to enable a retrieval strategy may learn new associations more quickly. Moreover, strategic reliance on memory retrieval should help to promote learning rates (Allen, Mahler, \& Estes, 1969). Conversely, older adults' avoidance of a retrieval strategy should delay learning new associations and, hence, rates of skill acquisition (see Rogers et al., 2000).

From a strategy shift affordance perspective, age differences in cognitive abilities could affect the objective cost/benefit ratio for strategy shift. Older adults might shift to retrieval use more slowly than do young adults because of greater shift cost (i.e., retrieval could be more time consuming or more error prone; see, e.g., Reder, Wible, $\&$ Martin, 1986) or lower shift benefit (i.e., the response time [RT] discrepancy between strategies could be smaller). Age differences might also exist in subjective influences on the cost/benefit analysis, such as beliefs about the relative difficulty and probable success for each strategy.

A useful way of addressing this issue is to manipulate the processing demands of the task in such a way as to manipulate the relative costs and benefits of the retrieval strategy and the rule-based strategy and then observe the effect on shift behavior. The construct of adaptivity in strategic behavior (Schunn \& Reder, 2001) relies on the assumption that individualscan and will adjust strategies to maximize benefit in changing task contexts.

There is some evidence that task affordances for different strategies may affect age differences in strategy shift. In the digit symbol substitution task (Wechsler, 1981), young adults typically outperform older adults with either a rule-based or a retrieval-based strategy (Erber, 1976). However, Cerella et al. (1999) demonstrated a distinct pattern of age differences with a simplified digit symbol task with a reduced display size. Older adults shifted to retrieval and learned the new pairings. Young adults, who were able to scan rapidly, did not learn the pairings or change strategies. This finding is consistent with the shift affordance perspective: Individuals will not shift to a retrieval-based process when the rule-based alternative is efficient.

We studied strategy shift in the noun pair look-up task. In this task, individuals search an array of word pairs to determine whether two target nouns are paired with each other in the array. Ackerman and Woltz (1994) studied two versions of the task, one in which the pairings in the array are variably mapped from trial to trial and one in which the nouns are consistently paired. In the varied version, participants must search the array to determine whether the target nouns are paired. In the consistent version, participants may respond either by scanning the array or by recognition memory for the pairings. A strategy shift typically occurs, with the more efficient retrieval strategy replacing the more costly scanning strategy. Ackerman and Woltz demonstrated that manipulation of task characteristics influenced the rate of the strategy shift.
Rogers et al. (2000) demonstrated dramatic age differences in noun pair strategy shift (see also Rogers \& Gilbert, 1997). Virtually all of their younger participants shifted to memory retrieval by the end of extended practice; however, $37 \%$ of their older sample continued to scan the array after extensive practice.

These studies suggest that some older adults may be averse to shifting to the use of the memory retrieval strategy. A possible explanation for this aversion is that older adults often lack confidence in their memory ability (e.g., Lineweaver \& Hertzog, 1998; see Hertzog \& Hultsch, 2000 , for a review) and, consequently, may attach greater subjective costs to the retrieval strategy. Older adults often sacrifice speed for accuracy in RT tasks (Hertzog, Vernon, \& Rypma, 1993; Ratcliff, Thapar, \& McKoon, 2001; Salthouse, 1979; Strayer \& Kramer, 1994). Therefore, they may choose to scan the noun pair array rather than to risk a rapid but incorrect retrieval-based response.

Our goal in this study was to examine the effect of task affordances for different strategies on age differences in strategy shift in the noun pair task. Scanning difficulty was manipulated by varying the display size (6 or 18 noun pairs); memorization difficulty was manipulated by varying the memory set size (6 or 18 noun pairs). Strategy shift was expected to be more rapid and more complete in a task for which shift is highly efficient (such as high scanning load with low memory load), as compared with a task for which shift is less efficient (such as low scanning load with high memory load).

Age differences in objective task performance and in subjective memory ability confidence were expected to influence how shift affordance affects strategy use. Furthermore, we hypothesized that older adults would be less likely to use retrieval strategies than would be warranted by their performance on memory tests (i.e., responding to a target pair without the opportunity to view the full array of possible pairings). We expected age differences in strategy shift to be associated with age differences in the rated confidence that individuals have in the use of the memory retrieval strategy.

A modification of strategy shift via manipulation of objective task affordances could be consistent with either instance theory (Logan, 1988) or the CMPL model (Rickard, 1997). Rickard's CMPL model can, in addition, account for subjective influences as the mechanism for strategy selection is not fully specified. The prediction that older adults will persist in rule-based processing despite the opportunity to rely on more efficient retrieval processes is unique to a shift affordance account and provides a strong test for our perspective.

\section{METHOD}

\section{Design}

The between-subjects independent variables were age (young or older), memory load, and scanning load. The full factorial combination of memory load ( 6 or 18 noun pairs) and scanning load (6 or 18 noun pairs) resulted in four shift affordance conditions: low scan- 
ning load with low memory load, low scanning load with high memory load, high scanning load with low memory load, and high scanning load with high memory load.

The different shift affordance conditions were accomplished as follows. In the conditions with equal scanning load and memory load, each noun pair was presented once in the look-up display. In the condition with low scanning load and high memory load, the array of noun pairs presented in the display (other than the target pair, which was necessarily presented) was randomized, with the qualification that all pairs were shown an equal number of times. In the condition with high scanning load and low memory load, the pairs were repeated either three or four times within the display (other than the target pair, which was presented only once), again with the qualification that all pairs were shown an equal number of times. Equal numbers of young and older adults were randomly assigned to each condition.

Comparison of the mixed load conditions is perhaps most relevant to the study of shift affordance. We expected that the participants given a low scanning load with a high memory load would have the lowest shift affordance and that the participants given a high scanning load with a low memory load would have the highest shift affordance. These condition differences are illustrated in Figure 1.

The within-subjects independent variables were task type (consistent mapping, memory test, or varied mapping), trial type (matched or unmatched), and practice (Repetition 1-120). The dependent variables were RTs, strategy reports, and percentage correct.

\section{Participants}

Eighty young adults between the ages of 18 and 23 years and 80 older adults between the ages of 60 and 75 years participated in the study. The young adults were recruited from the Georgia Institute of Technology School of Psychology participant pool, and the older adults were recruited from the Atlanta community by using the Adult Cognition Laboratory participant registry. The older adults had to be sufficiently healthy and intact to visit the laboratory on the Georgia Tech campus. The young adults received course credit for their participation, and the older adults received an honorarium $(\$ 10-15 / \mathrm{h})$ for their participation.

Prior to testing, the participants completed a demographic questionnaire and a short cognitive battery for the purpose of describing the age samples. The obtained age differences were comparable with those typically reported in the literature. The mean scores for these measures and the demographic characteristics of the participants in this experiment are summarized in Table 1.

\section{Procedure}

A computer controlled both stimulus presentations and the recording of the participants' responses. Timing accuracy to the near-

\begin{tabular}{|c|c|c|c|c|c|}
\hline VOICANO & GI_ACIER & BARREI. & POTATO & CAT & CAT \\
\hline DOLL & SE $\Lambda \mathrm{T}$ & $\mathrm{ST} \Lambda \mathrm{R}$ & FROG & MARKET & MARKET \\
\hline CAT & VOLCANO & BARREL & GLACIER & CAT & POTATO \\
\hline MARKEI & DOLL & STAR & SEAT & MARKET & FROG \\
\hline IVY & BARREL & РОТАТО & VOLCANO & $\mathrm{CAT}$ & GL $\Lambda$ CIER \\
\hline BIRD & STAR & ГROG & DOLL & MARKET & SEAT \\
\hline \multicolumn{6}{|c|}{$\begin{array}{c}\text { IVY } \\
\text { BIRD }\end{array}$} \\
\hline \multicolumn{6}{|c|}{ Is this pair of words matched? } \\
\hline & & Yes $=Y$ & $x_{0}=\mathrm{N}$ & & \\
\hline
\end{tabular}

\begin{tabular}{|c|c|c|c|c|c|}
\hline $\begin{array}{l}\text { STRING } \\
\text { IRON }\end{array}$ & $\begin{array}{l}\text { VOLCANO } \\
\text { DOLL }\end{array}$ & $\begin{array}{l}\text { BLOSSOM } \\
\text { HOOF }\end{array}$ & $\begin{array}{l}\text { BARREL } \\
\text { STAR }\end{array}$ & $\begin{array}{l}\text { BOWL } \\
\text { JURY }\end{array}$ & $\begin{array}{l}\text { LUMP } \\
\text { MULE }\end{array}$ \\
\hline \multicolumn{6}{|c|}{$\begin{array}{c}\text { BARREL } \\
\text { STAR }\end{array}$} \\
\hline \multicolumn{6}{|c|}{ Is this pair of words matched? } \\
\hline & & $Y c s=Y$ & $\mathrm{No}=\mathrm{N}$ & & \\
\hline
\end{tabular}

Figure 1. Sample trial screens used for the high shift affordance condition (top) and the low shift affordance condition (bottom). In the high shift affordance condition, 6 pairs were repeated in the 18-pair display. In the low shift affordance condition, a random 6 of the 18 total pairs were presented in the 6-pair display on a given trial. 
Table 1

Means (and Standard Deviations) for Measures of the Characteristics of the Research Participants

\begin{tabular}{lccccc}
\hline & \multicolumn{2}{c}{ Young } & & \multicolumn{3}{c}{ Old } \\
\cline { 2 - 3 } \cline { 5 - 6 } \multicolumn{1}{c}{ Measure } & $M$ & $S D$ & & $M$ & $S D$ \\
\hline Age (years)* & 19.4 & 1.33 & & 68.9 & 4.38 \\
Education* & 15.08 & 2.25 & & 13.57 & 1.28 \\
Health & 1.72 & 0.75 & & 1.96 & 0.86 \\
Limitations* & 1.16 & 0.53 & & 1.65 & 0.98 \\
Vocabulary* & 31.0 & 3.35 & & 35.6 & 3.74 \\
Digit symbol* & 74.83 & 12.39 & & 50.75 & 11.85 \\
First/last names* & 20.04 & 6.96 & & 9.91 & 5.27 \\
\hline
\end{tabular}

Note-Education, self-reported number of years of formal education (older adults reported higher mean years of completed education than did young adults; note, however, that the young adults were all college students and had not yet completed their education); health, selfreported using a scale from 1 (excellent) to 5 (poor); limitations, selfreported number of health-related limitations; digit symbol, score on WAIS-R digit symbol substitution subtest (Wechsler, 1981); first/last names, ETS first and last names subtest. $\quad * p<.01$

est millisecond was achieved by a routine that directly accessed the PC's internal clock. The participants were first trained on the noun pair look-up task. The participants then completed consistent training, followed by a block of memory test trials and a block of varied trials. Although the noun pair matches did not change in the consistent portion of the task, each noun pair location was randomly determined for each trial. Half of the trials presented a target noun pair that was matched in the look-up table, and half presented a target noun pair that was not matched in the look-up table. Unmatched pairs contained a top word paired with a randomly determined (with replacement) bottom word. We instructed the participants to press a key labeled "Y" if the target pair was matched in the look-up table or a key labeled " $\mathrm{N}$ " if the target pair was not matched in the lookup table. To explicitly measure strategy shift, strategy probes were included following each consistent trial. The participants were instructed to indicate which strategy they had used on the previous trial-(1) scan, (2) memory, (3) both, or (4) other-by pressing a key labeled with the first letter of the response strategy. ${ }^{1}$

Across consistent training, all the participants responded to 120 repetitions per stimulus. For the participants in the low memory load conditions, consistent training consisted of 20 presentation blocks in each of two sessions. Each block contained 18 trials, with three target repetitions each for the six noun pair stimuli. For the participants in the high memory load conditions, consistent training consisted of 30 blocks in each of four sessions. Each block contained 18 trials, with one target repetition each for the 18 noun pair stimuli. Across training, the participants responded to 120 repetitions per stimulus. The participants were offered a rest break following each block, during which they received feedback on their mean RT and accuracy for that block. We instructed the participants to keep their accuracy at $94 \%$, which corresponds to one error per block. If the participants' accuracy was below 94\%, they were asked to slow their responding; if the participants' accuracy was above $94 \%$, they were asked to speed their responding.

A transfer memory test block followed consistent training to measure noun pair learning. The participants completed one block (18 trials) of training without the noun pair look-up table. A transfervaried test block followed the memory test block to measure scanning speed. The participants completed one block (18 trials) of training with a display key with changing noun pair matchings on each trial. Because the noun pairings could not be memorized, the participants were required to scan the display on each trial. After the computer testing, the participants completed a posttest survey and debriefing. We asked the participants questions about strategy use and collected the following self-ratings (on a scale from 1 to 5) regarding the memory-based response strategy: confidence ("Were you confident to use your memory? $1=$ yes, $5=$ no"), effort ("How much effort did it require for you to memorize the word pairs? $1=$ automatic, $5=$ effortful"), and perceived improvement ("How much does using memory improve performance on this task? $1=$ very much, 5 = not at all").

\section{RESULTS}

\section{Consistent Performance}

We tested for differences in performance improvements across groups using mixed model analyses of RTs and accuracy data for the consistent noun pair trials (using SAS PROC MIXED; see Littell, Milliken, Stroup, \& Wolfinger, 1996). The model included age, memory load, scanning load, and repetition (continuous from 1 to 120) as fixed effects and person variation as a random effect. ${ }^{2}$

Response time improvements. A two-parameter power function of the form $\mathrm{RT}=b N^{-c}$ provides a good fit to the decrease in correct RTs with the number of repetitions per stimulus $(N)$. The parameters of the equation, $b$ and $c$, represent initial (baseline) performance and rate of change. After both sides of the equation are log transformed, it reduces to the linear relation $\log (\mathrm{RT})=b c *$ $\log (N)$ (see, e.g., Newell \& Rosenbloom, 1981). All analyses of RT were based on the median correct response latency for each participant. ${ }^{3}$

RTs by group and repetition are presented in Figure 2 . The young adults were faster than the older adults were $[F(1,6222)=306.53, p<.01 ; r=.68] .{ }^{4}$ Primarily owing to early performance levels, the individuals in the low scanning load conditions were faster than the individuals in the high scanning load conditions $[F(1,6222)=444.63$, $p<.01 ; r=.06]$. The RT difference between scanning load conditions was larger for the young adults $(r=.11)$ than for the older adults $(r=.06)$, resulting in a significant age $\times$ conditioninteraction $[F(1,6222)=10.13, p<$ $.01]$. Primarily owing to late performance levels, the individuals in the low memory load conditions were faster than were the individuals in the high memory load conditions $[F(1,6222)=5.44, p=.02 ; r=.17]$. The RT difference between memory load conditions was larger for the older adults $(r=.26)$ than for the young adults ( $r=$ .21 ), resulting in a significant age $\times$ condition interaction $[F(1,6222)=98.02, p<.01]$.

The interaction of scanning load and memory load was only marginally reliable $[F(1,6222)=3.62, p=.06]$. For the older adults, the difference in RTs between memory load conditions was larger in the low scanning load condition $(r=.31)$ than in the high scanning load condition $(r=.23)$. This trend was reversed for the young adults ( $r=.17$ and $r=.24$, respectively), resulting in a significant interaction of age, scanning load, and memory load $[F(1,6222)=34.12, p=.01]$.

Both the young and the older adults improved as a function of repetitions $[F(1,232)=2,577.80, p<.01]$. More absolute improvement occurred for the young adults than 


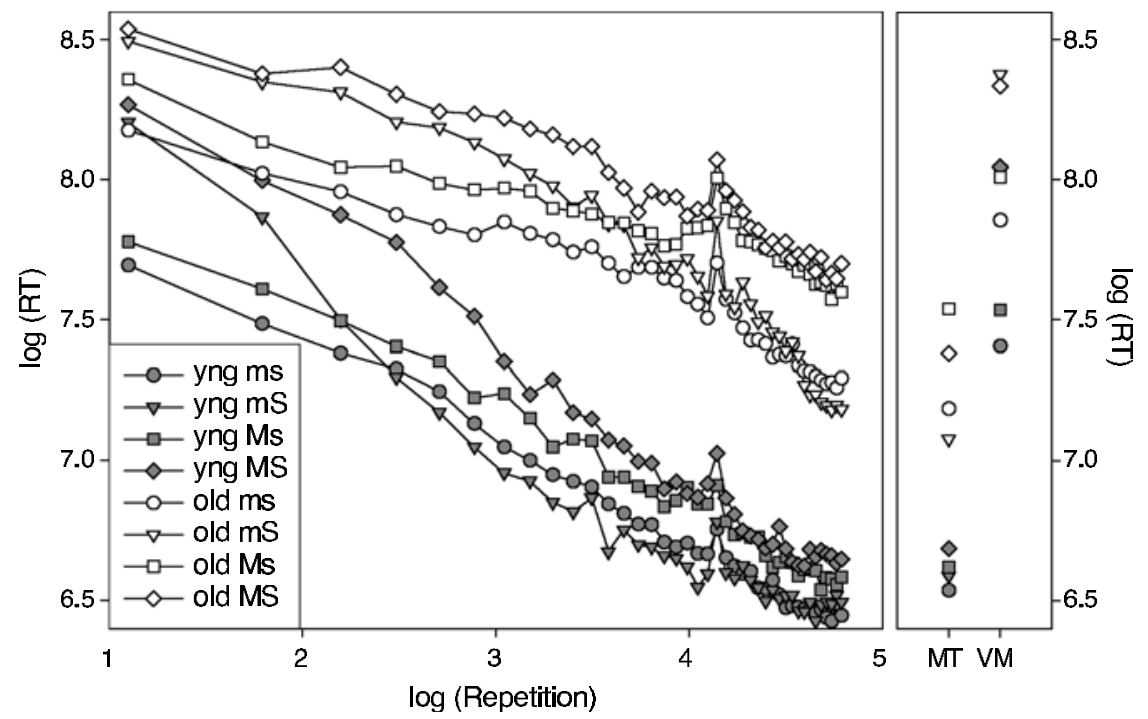

Figure 2. Response times (RTs) by age, shift affordance condition, and repetition for consistent training, memory test transfer, and varied transfer. In the legend, low memory load is represented by lowercase $\mathrm{m}$, high memory load is represented by uppercase $M$, low scanning load is represented by lowercase $s$, and high scanning load is represented by uppercase S. MT, memory test; VM, varied mapping.

for the older adults $[F(1,232)=101.53, p<.01]$. Owing to long RTs early in practice, more absolute improvement occurred for the high scanning load condition than for the low scanning load condition $[F(1,232)=56.29, p<.01]$. Owing to short RTs late in practice, more absolute improvement occurred for the low memory load condition than for the high memory load condition $[F(1,232)=11.47$, $p<.01]$. The improvement difference between memory load conditions was more pronounced for the older adults than for the young adults, resulting in a significant age $X$ memory load $\times$ repetitions interaction $[F(1,232)=24.37$, $p<.01]$. The age $\times$ scanning load $\times$ repetitions interaction $(p>.4)$ and the memory load $\times$ scanning load $\times$ repetitions interaction $(p>.8)$ were not significant.

The four-way interaction of age, memory load, scanning load, and repetition was also significant $[F(1,232)=$ $4.11, p<.05]$. This four-way interaction can be understood as follows. With training, the primary variable influencing RTs changed, with early RTs influenced most by scanning load and late RTs influenced most by memory load. Early RT separation (determined by scanning load) was larger for the young adults, whereas late RT separation (determined by memory load) was larger for the older adults. The change in the relative influence of these variables, which reflects strategy shift, occurred later in training for the older adults than for the young adults. Figure 2 illustrates the shift from scanning load influence to memory load influence as RTs cross over for the mixed load conditions (high scanning load with low memory load and low scanning load with high memory load).

Collectively, these results indicate that there was a greater separation of RT improvements by condition in the older adult groups, as well as an age difference in the rank order of RT by condition after extensive practice. The older adults also demonstrated slower rates of performance improvements, but their rates of improvement varied as a function of shift affordance. Most critical for our hypotheses, the older adults showed the greatest RT improvements in the high shift affordance condition, in which the benefit of shifting from scanning to retrieval should be (and was) the largest.

Accuracy data. For all analyses of accuracy data, probit transformation allowed for group comparisons despite universally high performance. A probit transformation returns the $p$ th quantile from the standard normal distribution, where $p$ is a probability between 0 and 1. Both age groups performed close to the $94 \%$ accuracy instruction, although the older adults $\left(M_{\text {raw }}=96.3, S D=4.9\right)$ were consistently more accurate than were the young adults $\left(M_{\text {raw }}=93.6, S D=7.1\right)[F(1,6221)=213.18, p<.01$; $r=.22]$. Accuracy was slightly higher in the low scanning load conditions than in the high scanning load conditions $[F(1,6221)=4.43, p=.04 ; r=.03]$. No interactions with the age or scanning load variables were noted, and accuracy was not affected by memory load or repetitions, as is seen by the absence of additional main effects or interactions $(p s>.06)$. In general, the high levels of accuracy pose few interpretational problems for analyzing performance improvements in RT.

Strategy shift. Reported proportions of trials using memory retrieval are presented in Figure 3. These data were also portrayed and analyzed in log-transformed coordinates. A mixed model analysis (identical to the consistent RT model above) was performed. The young adults re- 


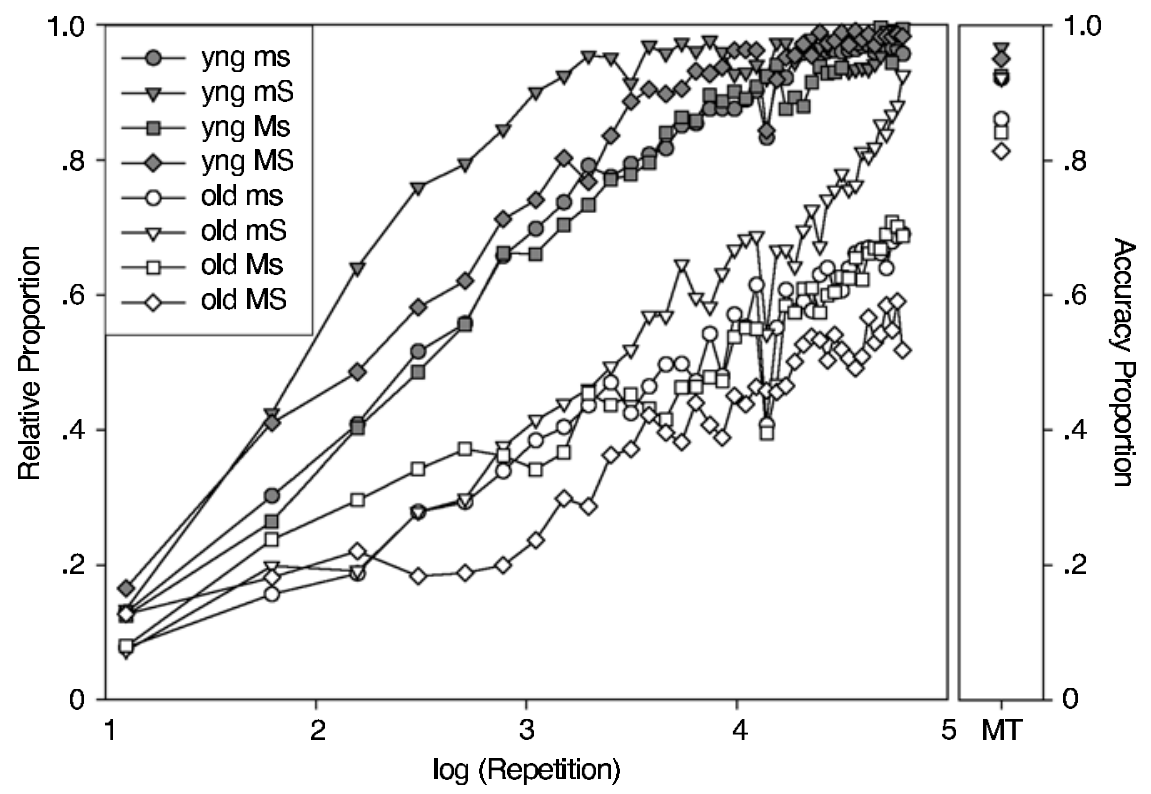

Figure 3. Left panel: Mean proportion of retrieval strategy use by age, shift affordance condition, and repetition for consistent training. Right panel: Mean accuracy proportion by age, shift affordance condition, and repetition for memory test transfer. In the legend, low memory load is represented by lowercase $m$, high memory load is represented by uppercase $M$, low scanning load is represented by lowercase $s$, and high scanning load is represented by uppercase S. MT, memory test.

ported a retrieval strategy more often than the older adults $\operatorname{did}[F(1,6222)=142.07, p<.01 ; r=.45]$. The individuals in the high scanning load conditions reported retrieval more than the individuals in the low scanning load conditions did $[F(1,6222)=15.85, p<.013 ; r=.04]$, with a difference that was larger for the young adults $(r=$ .11) than for the older adults $(r=.01)[F(1,6222)=$ $57.49, p<.01]$. The difference in retrieval reporting between the low and high memory load conditions was not significant $(p=.73)$. However, a larger memory load effect occurred for the older adults $(r=.12)$ than for the young adults $(r=.04)$, resulting in a significant age $\times$ memory load interaction $[F(1,6222)=30.35, p<.01]$.

The interaction of age, scanning load, and memory load $[F(1,6222)=9.64, p<.01]$ was significant and can be interpreted as follows. For the older adults, the difference in retrieval between memory load conditions was markedly larger in the high scanning load condition $(r=.25)$ than in the low scanning load condition $(r=.01)$. This trend was tempered for the young adults $(r=.07$ and $r=.01$, respectively). The interaction of scanning load and memory load was not significant ( $p=.27$ ).

Both the young and the older adults increased retrieval reports as a function of repetition $[F(1,265)=1,244.36$, $p<.01]$. More improvement occurred for the younger adults than for the older adults $[F(1,265)=5.64, p=.02]$. There was no significant difference in improvement between scanning load conditions or memory load conditions, nor was the interaction of scanning and memory load significant ( $p \mathrm{~s}>.2)$. However, the improvement difference between scanning load conditions was larger for the young adults than for the older adults, resulting in a significant age $\times$ scanning load $\times$ repetitions interaction $[F(1,265)=9.61, p<.01]$. As with the RT data, the improvement difference between memory load conditions was larger for the older adults than for the young adults, resulting in a significant age $\times$ memory load $\times$ repetitions interaction $[F(1,265)=13.60, p<.01]$.

The four-way interaction of age, memory load, scanning load, and repetition was significant $[F(1,265)=6.32$, $p<.02]$. This four-way interaction can be understood as follows. For the young adults, increases in retrieval reporting were primarily influenced by scanning load. For the older adults, increases in retrieval were influenced by the interaction of memory load and scanning load. To demonstrate this interaction more clearly, older adults' retrieval data for the last five repetitions were compared across memory load and scanning load groups. The memory load $\times$ scanning load interaction was significant $[F(1,265)=$ $13.60, p<.01]$. Under high scanning load, retrieval was greater for low memory load than for high memory load $(r=.43)$. Under low scanning load, however, there was no difference in retrieval use between memory load conditions $(r=-.02)$.

In summary, the retrieval report data strongly support the hypothesis that the high shift affordance condition resulted in an elevated use of retrieval strategies, relative to other conditions, for the older adults. Only in this condi- 
tion did the retrieval use of the older adults begin to approximate the uniformly high levels of retrieval strategy used by the younger adults.

\section{Memory Test Performance}

Immediately following consistent training, the participants were given a single block of memory test trials. The memory test trials were identical to the consistent trials, except that the look-up table was not shown at the top of the screen. We used mixed model analyses to compare memory test performance across age groups, scanning load, and memory load.

Response times. RTs are presented in the right-hand panel of Figure 2. Young adults' retrieval was faster than was older adults' retrieval $[F(1,152)=216.76, p<.01$; $r=.73]$, and the individuals in the high memory load condition were faster than those in the low memory load condition $[F(1,152)=20.09, p<.01 ; r=.22]$. These effects were qualified by an age $\times$ memory load interaction $[F(1,152)=6.44, p=.01]$, owing to an RT difference between memory load conditions that was larger for the older adults $(r=.40)$ than for the young adults $(r=.22)$. The main effect of scanning load was not significant $(p=$ .43). Older adults' retrieval was faster in the low scanning load condition than in the high scanning load condition $(r=.16)$, whereas the trend was reversed for young adults $(r=-0.14)$, resulting in a significant age $\times$ scanning load interaction $[F(1,152)=4.27, p=.04]$. All other memory test RT comparisons were nonsignificant ( $p \mathrm{~s}>.4$ ).

Accuracy data. Memory test accuracy (hits - false alarms) is presented in the right-hand panel of Figure 3. In order to facilitate comparison with retrieval proportions, we did not transform recognition memory within the figural presentations. In order to evaluate differences in recognition memory, we computed $d^{\prime}[z$ (hit rate) $-z$ (false alarm rate)]. The young adults were more accurate $\left(d^{\prime}=\right.$ $3.75)$ on the memory tests than were the older adults $\left(d^{\prime}=\right.$ 3.66) $[F(1,274)=12.73, p<.01 ; r=.21]$. Items that were reported as retrieved on the last block of consistent training had higher memory test accuracy than did items that were reported as scanned on the last block of consistent training $[F(1,274)=13.82, p=.02 ; r=.23]$. Although memory test accuracy was lower for recently scanned noun pairs than for recently retrieved noun pairs, the $86 \%$ accuracy rate was still notably high and well above chance. The age $\times$ reported strategy interaction was not significant ( $p=.14$ ). Memory test accuracy did not differ by memory load or scanning load, and all interactions with the load variables were nonsignificant $(p s>$ .08 ). Thus, actual learning of the noun pairs was not affected by the shift affordance condition, in contrast to the older adults' use of the memory retrieval strategy.

\section{Varied Performance}

Response times. The varied noun pair block provided an estimate of visual scanning times for the younger and the older adults, after extended consistent practice had allowed the individuals to learn general task characteristics.
RTs for the varied test are presented in the right-hand panel of Figure 2. We used a mixed model analysis to compare RTs across age group, scanning load, and memory load. The young adults were faster than were the older adults $[F(1,152)=110.66, p<.01 ; r=.46]$. As was expected, the individuals in the high scanning load condition were slower than were those in the low scanning load condition $[F(1,152)=182.15, p<.01 ; r=.31]$. The RT difference between high scanning load and low scanning load was larger for the young adults $(r=.80)$ than for the older adults $(r=.58)$, resulting in a significant age $\times$ scanning load interaction $[F(1,152)=4.08, p<.05]$.

No difference in scanning RTs was observed between the high and low memory load conditions $(p>.1)$, and memory load did not interact with age $(p>.8)$. Memory load did interact with scanning load, however $[F(1,152)=$ $4.60, p<.04$ ], owing to an RT difference between memory load conditions that was larger under low scanning load $(r=.28)$ than under high scanning load $(r=.03)$. For the individuals in the low memory load condition, improvements in search efficiency could occur for specific words repeated within the block of trials. The individuals in the low memory load conditions were presented with fewer individual words (12 nouns, corresponding to the six noun pairs in consistent training) than were those in the high memory load condition (36 words). Hence, each word was presented three times in the low memory load condition but only once in the high memory load condition. The resulting memory load difference in scanning RTs was manifested in the low scanning load conditions but was apparently overwhelmed by the greater difficulty of the high scanning load. The three-way age $\times$ memory load $\times$ scanning load interaction was not significant $(p>.6)$.

Accuracy data. Scanning accuracy was not affected by age, memory load, or scanning load, as was shown by the absence of significant main effects or interactions $(p \mathrm{~s}>.06)$.

\section{Response Time Confirmation of Strategy Reports}

One important feature of Figure 2 is that varied mapping (scanning) RTs, which were collected after consistent mapping practice, approximate initial consistent mapping RTs. Likewise, memory test RTs are close to consistent mapping RTs at the end of practice. These data suggest that the bulk of RT improvements in the noun pair task are associated with the strategy shift, not with RT improvements when scanning or retrieving. To analyze this facet of the data further, we will report consistent mapping noun pair RT improvements separately for the scanning and retrieval strategy reports.

The use of the other strategy was minimal during consistent testing, occurring in approximately $0.5 \%$ of the responses. Use of the both strategy was somewhat more frequent, occurring in approximately $7.6 \%$ of the responses. If use of the both strategy is interpreted as an indication of verification behavior (i.e., a retrieval followed by a visual 
search to verify the retrieved answer), then RTs for the both strategy should be more similar to scanning RTs than to retrieval RTs.

Comparison of strategy RTs also provided assurance that strategy reports accurately reflected participant behavior. RTs should have been faster on trials with reported memory retrieval. A mixed model analysis compared RTs for the different strategy reports across age groups and strategy report (retrieval, scan, or both). There was a reliable main effect of strategy report $[F(2,436)=100.16$, $p<.01]$. Focused comparisons showed that retrieval RTs were indeed faster than were scanning RTs $[t(436)=$
$-13.02, p<.01 ; r=-.62]$ and RTs for the both strategy reports $[t(436)=-11.01, p<.01 ; r=-.50]$, although no reliable difference was seen between RTs for the scanning strategy and RTs for the both strategy $(p=.29)$. The age group $X$ strategy report interaction was not significant $(p=.08)$. Given that trials with the both response were infrequent and appeared to behave in the same way as the scan report trials, we excluded them from further analysis.

For each age group in all conditions, scanning RTs were clearly longer than retrieval RTs. Figure 4 demonstrates this separation by plotting RTs for scanning reports in the
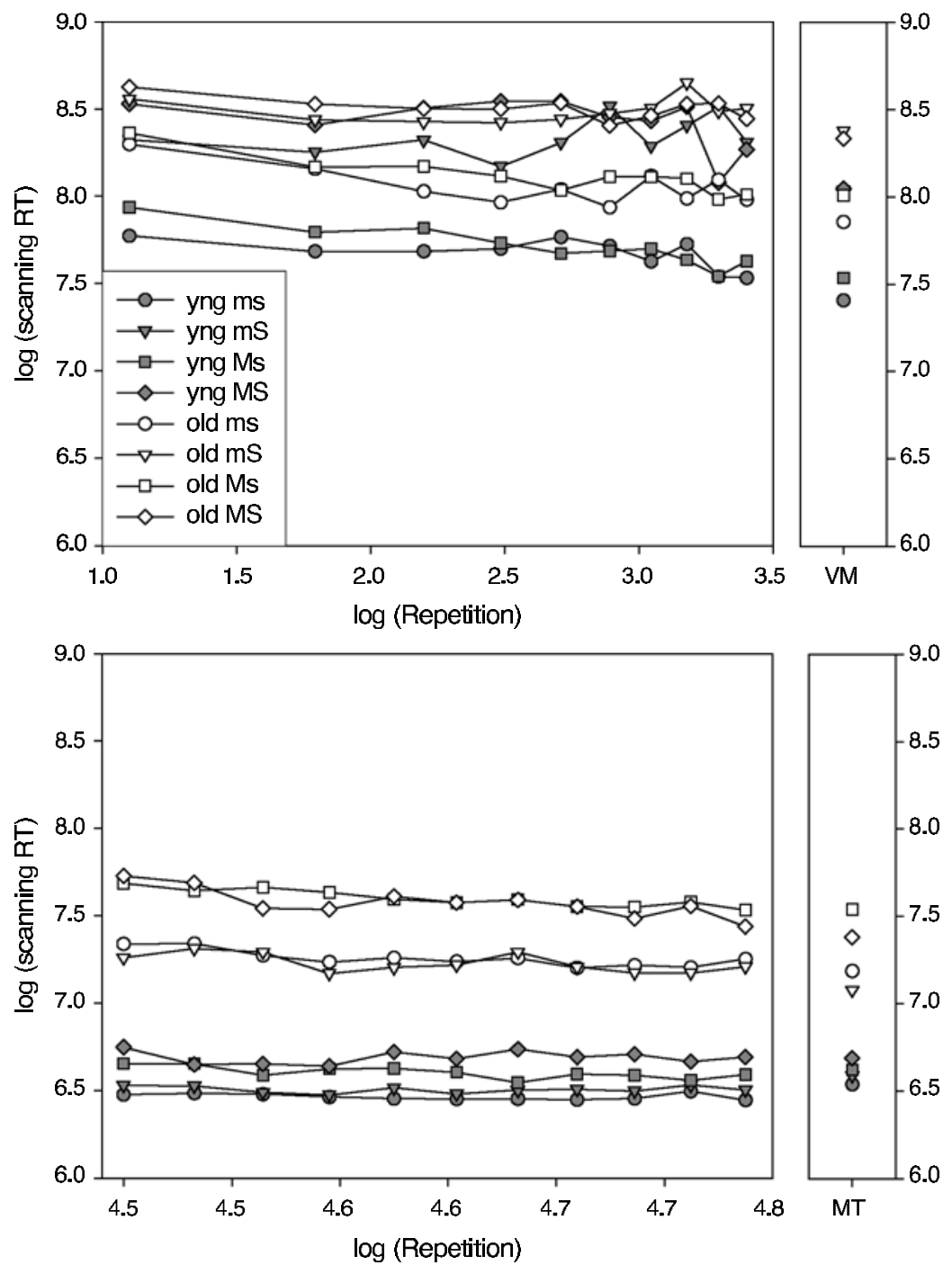

Figure 4. Top panel: Scanning response times (RTs) by age, shift affordance condition, and repetition for the first 10 blocks of consistent training and for the varied test block. Bottom panel: Retrieval response times by age, shift affordance condition, and repetition for the last $\mathbf{1 0}$ blocks of consistent training and for the memory test block. In the legend, low memory load is represented by lowercase $\mathbf{m}$, high memory load is represented by uppercase $M$, low scanning load is represented by lowercase $s$, and high scanning load is represented by uppercase S. VM, varied mapping; MT, memory test. 
first 10 blocks of training and RTs for retrieval reports in the last 10 blocks of training. 5 The pattern of strategy RTs supported the validity of the strategy report as an assessment of participant behavior. Note also that the performance improvements within types of strategy were relatively minor. Most of the variance in consistent mapping RT improvement was accounted for by the shift from scanning to retrieval.

To further demonstrate this phenomenon, Figure 4 also presents RTs for scanning from the varied testing block and RTs from the memory testing block. Scanning RT was measured at Block 10 of consistent training, because many of the participants (particularly the young adults) reported no scanning after this point, and improvements in scanning report RTs were minimal after this point. Retrieval RT was measured at the final block of consistent training. Scanning report RTs were equivalent to RTs in the varied testing block $(p>.1)$, and no interactions were found with the age or condition variables $\left(p_{\mathrm{s}}>.3\right)$. Retrieval report RTs were equivalent to RTs in the memory testing block $(p>.3)$, and no interactions were found with the age or condition variables ( $p \mathrm{~s}>.4$ ). These results, coupled with the comparison of RTs across strategies, offer strong support for the validity of the strategy reports.

\section{Posttest Ratings}

Posttest ratings were examined in age $\times$ condition analyses of variance (ANOVAs). The older adults rated memorizing the word pairs as more effortful than did the young adults $\left[M_{\text {old }}=3.26, S D_{\text {old }}=0.89 ; M_{\text {young }}=2.31\right.$, $\left.S D_{\text {young }}=0.93 ; F(1,145)=43.43, p<.01 ; r=.46\right]$. The older adults gave lower ratings to the memory strategy as a means of improving performance $\left[M_{\text {old }}=1.91, S D_{\text {old }}=\right.$ $0.95 ; M_{\text {young }}=1.15, S D_{\text {young }}=0.39 ; F(1,146)=42.25$, $p<.01 ; r=.46]$. The older adults were also less confident in their ability to rely on memory than were the young adults $\left[M_{\text {old }}=2.48, S D_{\text {old }}=1.11 ; M_{\text {young }}=1.30\right.$, $\left.S D_{\text {young }}=0.46 ; F(1,146)=75.06, p<.01 ; r=.57\right]$. In general, the older adults expressed less confidence in and more difficulty with the retrieval strategy, consistent with their less frequent use of that strategy.

\section{Confidence in Memory Retrieval}

Underconfidence in their memory ability might make the older adults less willing to rely on a retrieval-based strategy. Comparison of memory test block accuracy with the last block of strategy report data provides an indirect examination of memory retrieval confidence for the noun pair task. Since no additional training occurred between consistent training and the memory tests, the level of item knowledge for the memory test block was assumed to be unchanged from the last block of consistent training.

Figure 5 plots memory test accuracy as a function of retrieval use in the last block of consistent training by age and shift affordance condition. If the individuals had accurately monitored their item knowledge at the end of consistent training (see Dunlosky \& Hertzog, 2000), and

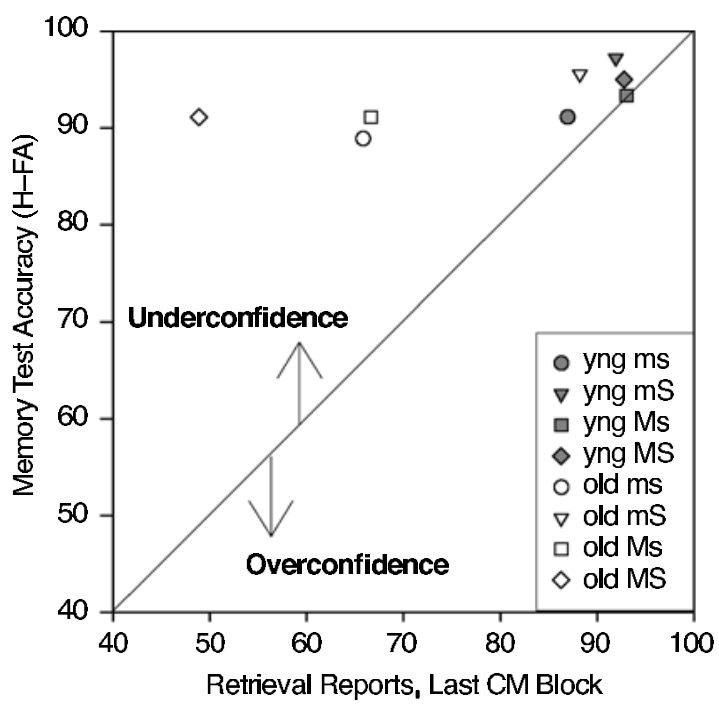

Figure 5. Mean memory test accuracy as a function of mean percentage of retrieval use for the last block of consistent training, by age and shift affordance condition groups. In the legend, low memory load is represented by lowercase $\mathrm{m}$, high memory load is represented by uppercase $M$, low scanning load is represented by lowercase $s$, and high scanning load is represented by uppercase S. H, hits; FA, false alarms; CM, consistent mapping.

if their strategy use was calibrated to their item knowledge awareness (see Keren, 1991), then in the aggregate we would expect good agreement between the two variables. If strategy use is calibrated to item knowledge, the data points should lie on or near the diagonal. Points above the diagonal indicate underconfident behavior, whereas points below the diagonal signify overconfident behavior. ${ }^{6}$ Figure 5 shows that the means for the young adults' conditions fall close to the diagonal. The means for the older adults' conditions deviate substantially from the diagonal, with the exception of the condition that affords strategy shift (large scanning load with small memory load).

One way to evaluate whether older adults are underconfident is to analyze the retrieval reports across age groups, controlling for memory test accuracy as a covariate. To the extent that retrieval reports merely recapitulate item learning, age differences and condition differences in use of the retrieval strategy should be eliminated by covarying on memory test accuracy. On the other hand, if confidence affects retrieval use, we would expect both variables to predict retrieval strategy use by older adults.

We ran a mixed model analysis to compare retrieval reports across age groups, with memory test accuracy as a covariate. The memory test covariate was significant $[F(1,155)=11.72, p<.01]$. As was shown earlier, retrieval reports were higher for the young adults than for the older adults $[F(1,155)=5.66, p<.02]$. This age difference was not eliminated when retrieval was covaried with memory test accuracy $[F(1,155)=5.82, p<.02]$. 


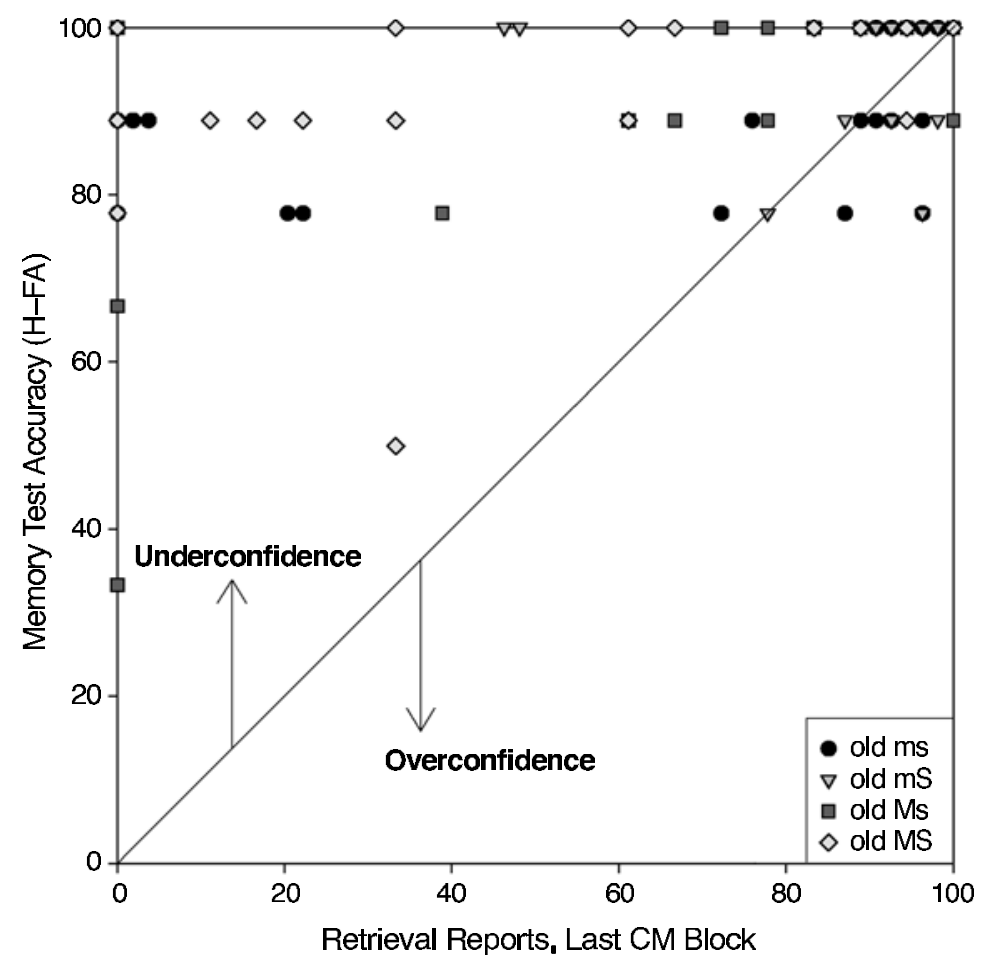

Figure 6. Mean memory test accuracy as a function of mean percentage of retrieval use for the last block of consistent training, for individual older adult participants by shift affordance condition. In the legend, low memory load is represented by lowercase $\mathrm{m}$, high memory load is represented by uppercase $M$, low scanning load is represented by lowercase $s$, and high scanning load is represented by uppercase $\mathrm{S}$. $\mathrm{H}$, hits; FA, false alarms; CM, consistent mapping.

These results provide further evidence for the claim that older adults' retrieval reluctance is not exclusively determined by low memory ability.

The phenomenon is also observable at the level of individual older participants (younger adults showed little variability in retrieval strategy use at the end of practice). Figure 6 plots older individuals' memory test accuracy and retrieval strategy use. A substantial number of older adults manifested major discrepancies between the two variables, with high memory test accuracy often associated with low probability of memory strategy use. To verify that this reluctance to shift is associated with individual differences in beliefs about the memory strategy, we computed three multiple regression analyses, one for each posttest rating variable. These regressions employed retrieval use at the end of practice as the dependent variable and memory accuracy and one of the three rating scales as independent variables. Ratings of confidence in the use of the memory strategy and memory test performance reliably predicted older adults' retrieval use in the noun pair task $\left[R^{2}=.25 ; F(2,73)=11.99, p>.01\right]$, with similar regression weights $(\beta=-.33$ for memory performance; $\beta=-.29$ for retrieval confidence; $p<.01$ ). Thus, memory for the pairings is not a sufficient account of retrieval strategy use; beliefs appear to have an independent influ- ence on strategic behavior. The identical analysis with the other rating variables also produced a significant regression coefficient for perceived improvement $(\beta=-.30$, $p<.01$ ) but not for rated effort in using the strategy ( $\beta=$ $-.17, p>.10)$. Self-reports of the ability to use and benefit from the retrieval strategy predicted noun pair retrieval use when actual recognition memory for the noun pairs was controlled for.

\section{DISCUSSION}

The present study replicates and extends previous research on age differences in rates of skill acquisition. Prior investigations of age differences in noun pair look-up performance indirectly measured strategy shift in terms of changes in RTs and comparisons of consistent and varied RT distributions (Rogers et al., 2000). A critical feature of the present study is the collection of strategy reports, which allowed us to directly track changes in strategic behavior (Rickard, 1997; Touron, Hoyer, \& Cerella, 2003). Our results confirm an age difference in the reliance on a memory retrieval strategy that is influenced by manipulation of shift affordance.

In general, failure to account for qualitative difference in processing strategies can lead to erroneous inferences 
about the cognitive mechanisms underlying learning (MacLeod, Hunt, \& Mathews, 1978; Schunn \& Reder, 2001). It can also distort estimates of the nature of the learning function (Delaney, Rader, Staszewski, \& Ritter, 1998; Rickard, 1997). The failure to separate an older sample into those who retrieve and those who scan results in distorted noun pair learning curves for the aggregate older sample.

Most of the performance improvements we observed were determined by a shift in strategies, not by improvements in the efficiency of scanning and retrieval processes. When strategy shift is not obligatory, both task factors (e.g., strategy demands) and person factors (e.g., confidence in memory ability) can affect strategy choice. Strategy shift affordance influenced the timing of strategy shift, particularly for the older adults. Young adult performance was most influenced by the scanning load benefit, whereas older adult performance was influenced by both the scanning load benefit and the memory load cost. The older adults in the highest shift affordance condition (high scanning load with low memory load) displayed greater willingness to rely on memory retrieval, as was manifested by the difference between memory test performance and retrieval strategy use, than did the older adults in lower shift affordance conditions.

It might be argued that what we consider a reluctance to shift strategy is instead an outcome of a pure associative learning deficit, with older adults shifting to retrieval slowly because of age-related impairments in the acquisition of associative recognition (Naveh-Benjamin, 2000). Undoubtedly, age differences in rates of associative learning influence these outcomes, but they cannot account for our findings. Given random assignment, learning ability should not differ by strategy affordance condition. Furthermore, the conditions provided equal numbers of repetitions for each noun pair. The timing of retrieval-based responding is determined by the number of item repetitions, which we equated, rather than by the number of items to be learned (Logan \& Klapp, 1991; see also Logan \& Etherton, 1994). Therefore, a finding of slower shift to retrieval in high memory load is unlikely to be caused by a simple associative learning deficit. In equating item repetitions, however, we were required to provide more training to individuals in the high memory load conditions. This additional training could have led to higher levels of fatigue and forgetting, and therefore could have increased the role of a learning deficit. A comparison of intersession intervals did not support this account. ${ }^{7}$ In addition, (1) the equivalence of memory test accuracy across memory load and scanning load conditions, (2) the interaction of memory load and scanning load influence for RTs, retrieval use, and difference scores, and (3) the obtained relationship between strategy shift reluctance and metacognitive reports of memory confidence all mitigate against a simple associative deficit hypothesis and support instead the contention that the slowed improvement of older adults in the noun pair task can be partially attributed to an aversion to shift from a scanning strategy.
Although our evidence favors the argument that age differences in strategy shift are influenced by subjective factors, one limitation of the present design was that we measured memory for the noun pairs only after the completion of the noun pair training. Rogers et al. (2000) did include interpolated memory test blocks throughout practice (with both free recall for the noun pairs and recognition memory tests, as in the present study). Including interim memory tests increases the rate of strategy shift for older adults (Rogers \& Gilbert, 1997) but does not eliminate the age difference in strategy shift. Nevertheless, future research could profitably focus specifically on whether older individuals are less likely to opt for the retrieval strategy during training even when memory probes show that they can successfully discriminate items on the basis of memory retrieval.

Older adults were less confident in their ability to achieve accurate performance by using a memory retrieval strategy. Additional research will be needed to determine the extent to which older adults' aversion to risk errors can be overcome by experimental manipulations and whether changing criteria would lead to faster strategy shifts for older adults in noun pair performance. Conservative response biases in healthy older adults might be fundamentally maladaptive, or they might represent a compensatory approach to normal cognitive decline with aging (Bäckman \& Dixon, 1995).

Both the instance model (Logan, 1988) and Rickard's (1997) CMPL model allow rule-based responding when memory retrieval is possible. Only CMPL, however, allows for rule-based processing when the rule RT distribution is slower than the retrieval RT distribution. On this count, our results appear to be more consistent with CMPL than with the instance model. Although scanning RTs were slower than were retrieval RTs across age group and noun pair task conditions, many older adults persisted in scanning behavior. In support of the affordance perspective is the fact that older adults in conditions with low shift affordance were especially likely to persist in rule execution despite having the potential for faster retrievalbased processing.

Although these findings can be accommodated by CMPL, that theory is agnostic with respect to the mechanisms by which a strategic choice is made. The present research provides no direct evidence regarding the nature of underlying choice mechanisms. One class of mechanisms involves an intentional decision to learn the pairings, resulting in the differential likelihood of using encoding strategies as a function of shift affordances. Individuals might differentially allocate effort to encoding on the basis of the subjective costs and benefits for the scanning and retrieval strategies. However, we observed no differences in noun pair memory between shift affordance conditions at the end of training, despite retrieval use differences. In addition, RTs in the present data were much shorter than would be expected for intentional encoding, given the time required to generate mediators for paired associates (e.g., Hertzog, Dunlosky, \& Robinson, 2003). 
A second class of candidate mechanisms consists of metacognitive processes involved in rapid decisions to retrieve or scan at the time of stimulus presentation. Rapid decisions to retrieve or scan could be based on the degree of feeling of knowing (FOK) the correct answer (e.g., Schunn et al., 1997). By this account, age differences in decisions to use the memory retrieval strategy could be caused by lower FOK levels or by a higher threshold criterion of FOK in the selection of a retrieval strategy. Marquié and Huet (2000) showed that older adults' lower memory self-efficacy beliefs (see Hertzog \& Hultsch, 2000) are associated with lower mean FOK levels for computer knowledge questions, relative to general knowledge questions. That study suggests that older adults may have lower confidence in their ability to retrieve answers from memory. Alternatively, older adults may have an impairment in FOK-monitoring accuracy, which limits discrimination of learned from nonlearned items. An impairment in monitoring accuracy could lead to routine reliance on the scanning strategy. Older adults are generally reported to have FOK accuracy equivalent to that of younger adults (e.g., Butterfield, Nelson, \& Peck, 1988; Marquié \& Huet, 2000), but virtually all the studies in the literature have used FOK for knowledge questions (i.e., items that tap retrieval from semantic memory). Souchay, Isingrini, and Espagnet (2000) argued that episodic FOK accuracy (as in the kind of list-learning experiment reported here), but not semantic FOK accuracy, is impaired in old age. In any case, an FOK-based account seems to require an additional mechanism that varies across shift affordance conditions to explain the strategy shift affordance effect. Augmenting the FOK mechanism with a changing criterion to select the retrieval strategy (i.e., a lower decision criterion in the high shift affordance condition) could, in principle, explain the pattern of results.

Strategy selection could also be based on the retrieval of a candidate answer and the subjective experiences that accompany that retrieval (Koriat \& Levy-Sadot, 2001). This kind of "slow" FOK appears to be based on cue accessibility (i.e., what is retrieved and how rapidly it is accessed). There are age differences in a variety of retrieval phenomena that could affect cue accessibility and that could also lower older adults' confidence in the accuracy of retrieved information (Zacks, Hasher, \& Li, 2000). In particular, work on associative recognition suggests that responses can be based on either recollection or familiarity experiences, as defined by a two-process model for recognition memory (e.g., Yonelinas, 2001). Older adults are believed to rely more on familiarity processes in recognition memory (e.g., Hay \& Jacoby, 1999; see Light, Prull, LaVoie, \& Healy, 2000, for a review). Familiarity is typically accompanied by lower levels of confidence in the candidate answer that has been retrieved (e.g., Yonelinas, 2001). Hence, older adults could correctly recognize items on the basis of familiarity but have lower confidence in the correctness of their responses. Furthermore, older adults' lower memory self-efficacy could also reduce confidence in retrieved answers directly, leading to an in- creased tendency to choose scanning over retrieving. Again, however, the shift affordance effect observed in this study would seem to indicate that older adults can flexibly adjust a confidence-based criterion for selecting the retrieval strategy. By this account, we might expect older adults to manifest lower confidence in the accuracy of retrieved answers-which would lead to reduced likelihood of strategy shift—but to be able to lower their retrieval selection confidence criterion when motivated to do so.

The alternatives we have highlighted certainly do not exhaust the set of candidate explanations, but they do seem a reasonable basis for further research on age differences in the rates and degree of strategy shift in the noun pair task.

\section{REFERENCES}

ACKERMAN, P. L., \& WOLTz, D. J. (1994). Determinants of learning and performance in an associative memory/substitution task: Task constraints, individual differences, volition, and motivation. Journal of Educational Psychology, 86, 487-515.

Allen, G. A., Mahler, W. A., \& Estes, W. K. (1969). Effects of recall tests on long-term retention of paired associates. Journal of Verbal Learning \& Verbal Behavior, 8, 463-470.

BÄCKMAN, L., \& DiXON, R. A. (EDS.) (1995). Compensating for psychological deficits and declines: Managing losses and promoting gains. Mahwah, NJ: Erlbaum.

Bosman, E. A., \& CHARnEss, N. (1996). Age differences in skilled performance and skill acquisition. In T. Hess \& F. Blanchard-Fields (Eds.), Perspectives on cognitive change in adulthood and aging (pp. 428-453). New York: McGraw-Hill.

Bourne, L. E., Healy, A. F., PArker, J. T., \& Rickard, T. C. (1999). The strategic basis of performance in binary classification tasks: Strategy choices and strategy transitions. Journal of Memory \& Language, 41, 223-252.

Butterfield, E. C., Nelson, T. O., \& Peck, V. (1988). Developmental aspects of the feeling of knowing. Developmental Psychology, 24, 654-663.

Cerella, J., Green, D. R., \& Hoyer, W. J. (1999, November). Effects of age and of item frequency on the memory and computational components of instance learning. Paper presented at the Annual Meeting of the Psychonomic Society, Los Angeles.

Charness, N., \& CAmpbell, J. I. D. (1988). Acquiring skill at mental calculation in adulthood: A task decomposition. Journal of Experimental Psychology: General, 117, 115-129.

Delaney, P. F., Reder, L. M., Staszewski, J. J., \& Ritter, F. E. (1998). The strategy-specific nature of improvement: The power law applies by strategy within task. Psychological Science, 9, 1-7.

Dunlosky, J., \& Hertzog, C. (2000). Updating knowledge about encoding strategies: A componential analysis of learning about strategy effectiveness from task experience. Psychology \& Aging, 15, 462474.

ERBER, J. T. (1976). Age differences in learning and memory on a digitsymbol substitution task. Experimental Aging Research, 2, 45-53.

ERICSSON, K. A., \& CHARNESS, N. (1994). Expert performance: Its structure and acquisition. American Psychologist, 49, 725-747.

HAY, J. F., \& JАСОвY, L. L. (1999). Separating habit and recollection in young and elderly adults: Effects of elaborative processing and distinctiveness. Psychology \& Aging, 14, 122-134.

Hertzog, C., Dunlosky, J., \& Robinson, A. E. (2003). Encoding fluency is a cue used for judgments about learning. Journal of Experimental Psychology: Learning, Memory, \& Cognition, 29, 22-34.

Hertzog, C., \& Hultsch, D. F. (2000). Metacognition in adulthood and old age. In F. I. M. Craik \& T. A. Salthouse (Eds.), The handbook of aging and cognition (2nd ed., pp. 417-466). Mahwah, NJ: Erlbaum.

Hertzog, C., Vernon, M. C., \& Rypma, B. (1993). Age differences in mental rotation task performance: The influence of speed/accuracy tradeoffs. Journals of Gerontology, 48, 150-156.

JENKINS, L., \& HOYER, W. J. (2000). Instance-based automaticity and 
aging: Acquisition, reacquisition, and long-term retention. Psychology \& Aging, 15, 551-565.

KAUSLER, D. H. (1994). Learning and memory in normal aging. San Diego: Academic Press.

Keren, G. (1991). Calibration and probability judgments: Conceptual and methodological issues. Acta Psychologica, 77, 217-273.

KORIAT, A., \& LEVY-SADOT, R. (2001). The combined contributions of the cue-familiarity and accessibility heuristics to feelings of knowing. Journal of Experimental Psychology: Learning, Memory, \& Cognition, 27, 34-53.

Light, L. L., Prull, M. W., LaVoie, D. J., \& Healy, M. R. (2000). Dual-process theories of memory in old age. In T. J. Perfect \& E. A. Maylor (Eds.), Models of cognitive aging (pp. 238-300). Oxford: Oxford University Press.

Lineweaver, T. T., \& Hertzog, C. (1998). Adults' efficacy and control beliefs regarding memory and aging: Separating general from personal beliefs. Aging, Neuropsychology, \& Cognition, 5, 264-296.

Littell, R. C., Milliken, G. A., Stroup, W. W., \& Wolfinger, R. (1996). SAS system for mixed models. Cary, NC: SAS Institute.

LogAN, G. D. (1988). Toward an instance theory of automatization. Psychological Review, 95, 492-527.

Logan, G. D., \& Etherton, J. L. (1994). What is learned during automatization? The role of attention in constructing an instance. Journal of Experimental Psychology: Learning, Memory, \& Cognition, 20, 1022-1050.

LOGAN, G. D., \& KLAPP, S. T. (1991). Automatizing alphabet arithmetic: I. Is extended practice necessary to produce automaticity? Journal of Experimental Psychology: Learning, Memory, \& Cognition, 17, 179195.

MacLeod, C. M., Hunt, E. B., \& Mathews, N. N. (1978). Individual differences in the verification of sentence-picture relationships. Journal of Verbal Learning \& Verbal Behavior, 17, 493-507.

MARquié, J. C., \& HuET, N. (2000). Age differences in feeling-ofknowing and confidence judgments as a function of knowledge domain. Psychology \& Aging, 15, 451-461.

NAVEH-BENJAMIN, M. (2000). Adult age differences in memory performance: Tests of an associative deficit hypothesis. Journal of Experimental Psychology: Learning, Memory, \& Cognition, 26, 1170-1187.

Naveh-Benjamin, M., Craik, F. I. M., GueZ, J., \& Dori, H. (1998). Effects of divided attention on encoding and retrieval processes in human memory: Further support for an asymmetry. Journal of Experimental Psychology: Learning, Memory, \& Cognition, 24, 10911104.

Newell, A., \& Rosenbloom, P. S. (1981). Mechanisms of skill acquisition and the law of practice. In J. R. Anderson (Ed.), Cognitive skills and their acquisition (pp. 1-56). Hillsdale, NJ: Erlbaum.

RATClifF, R., Thapar, A., \& MCKoON, G. (2001). The effects of aging on reaction time in a signal detection task. Psychology \& Aging, 16, 323-341.

REDER, M., \& RitTER, F. E. (1992). What determines initial feeling of knowing? Familiarity with question terms, not with the answer. Journal of Experimental Psychology: Learning, Memory, \& Cognition, 18, 435-451.

Reder, L. M., Wible, C., \& Martin, J. (1986). Differential memory changes with age: Exact retrieval versus plausible inference. Journal of Experimental Psychology: Learning, Memory, \& Cognition, 12, 72 81.

RICKARD, T. C. (1997). Bending the power law: A CMPL theory of strategy shifts and the automatization of cognitive skills. Journal of Experimental Psychology: General, 126, 288-310.

Rogers, W. A., \& GILBERT, D. K. (1997). Do performance strategies mediate age-related differences in associative learning? Psychology \& Aging, 12, 620-633.

Rogers, W. A., Hertzog, C., \& Fisk, A. D. (2000). An individual differences analysis of ability and strategy influences: Age-related differences in associative learning. Journal of Experimental Psychology: Learning, Memory, \& Cognition, 26, 359-394.

RoSENTHAL, R., \& Rosnow, R. L. (1991). Essentials of behavioral research: Methods and data analysis (2nd ed.). New York: McGrawHill.
Salthouse, T. A. (1979). Adult age and the speed-accuracy trade-off. Ergonomics, 22, 811-821.

SAlthouse, T. A. (1994). Aging associations: Influence of speed on adult age differences in associative learning. Journal of Experimental Psychology: Learning, Memory, \& Cognition, 20, 1486-1503.

SCHUNN, C. D., \& REDER, L. M. (2001). Another source of individual differences: Strategy adaptivity to changing rates of success. Journal of Experimental Psychology: General, 130, 59-76.

SChunN, C. D., Reder, L. M., Nhouyvanisvong, A., Richards, D. R., \& Stroffolino, P. J. (1997). To calculate or not to calculate: A source activation confusion model of problem familiarity's role in strategy selection. Journal of Experimental Psychology: Learning, Memory, \& Cognition, 23, 3-29.

SHIFFrin, R. M., \& SCHNEIdER, W. (1977). Controlled and automatic human information processing: II. Perceptual learning, automatic attending, and a general theory. Psychological Review, 84, 127-190.

Souchay, C., Isingrini, M., \& Espagnet, L. (2000). Aging, episodic memory, feeling-of-knowing, and frontal functioning. Neuropsychology, 14, 299-309.

Strayer, D. L., \& Kramer, A. F. (1994). Aging and skill acquisition: Learning-performance distinctions. Psychology \& Aging, 9, 589-605.

Touron, D. R., Hoyer, W. J., \& Cere lla, J. (2001). Cognitive skill acquisition and transfer in younger and older adults. Psychology \& Aging, 16, 555-563.

Touron, D. R., Hoyer, W. J., \& Cerella, J. (2003). Aging of item learning in cognitive skill acquisition. Manuscript submitted for publication.

WECHSLER, D. (1981). Wechsler Adult Intelligence Scale-Revised. New York: Psychological Corporation.

WENGER, M. J. (1999). On the whats and hows of retrieval in the acquisition of a simple skill. Journal of Experimental Psychology: Learning, Memory, \& Cognition, 25, 1137-1160.

YonElinAs, A. P. (2001). Consciousness, control, and confidence: The 3 Cs of recognition memory. Journal of Experimental Psychology: General, 130, 361-379.

ZACKS, R. T., HASHER, L., \& LI, K. Z. H. (2000). Human memory. In F. I. M. Craik \& T. A. Salthouse (Eds.), The handbook of aging and cognition (2nd ed., pp. 293-357). Mahwah, NJ: Erlbaum.

\section{NOTES}

1. Both was included to measure incidences in which participants scan despite having memorized the item, owing to slow recall or a need to verify. Other was included to measure incidences of guessing or uncertainty about strategy use.

2. A preliminary analysis was completed to test for differences between matched and unmatched trial types. Matched trials were found to have longer RTs and higher retrieval use than did unmatched trials $(p s<$ .01 ), but there were no interactions of trial type with age, memory load, scanning load, or repetitions. For that reason, data were pooled over the trial type factor for further analysis.

3. Median RTs were computed at the block level for each participant to reduce the influence of exceptionally long (owing perhaps to distractions) or short (owing perhaps to guessing) outliers.

4. Effect size correlations are provided as measures of comparison magnitudes independent of sample size. We have used the effect size correlation computed from Cohen's $d$, with difference standardized by pooled variance (see Rosenthal \& Rosnow, 1991).

5 . We did not include all blocks of training because of changing strategy proportions. Visual examination of the full data set supported the RT separation by strategy.

6. It is unlikely that memory test accuracy and retrieval probability are equivalently scaled, and hence, one should treat these criteria for underand overconfidence as approximate and heuristic.

7. Intersession comparisons in the high memory load condition were statistically indistinguishable from equality for both RTs $(p=.36)$ and retrieval proportions $(p=.27)$.

(Manuscript received June 3, 2002; revision accepted for publication September 19, 2003.) 